\title{
On some new inequalities for differentiable co- ordinated convex functions
}

\author{
MA Latif ${ }^{*}$ and SS Dragomir ${ }^{2}$
}

* Correspondence:

m_amer_latif@hotmail.com

${ }^{1}$ College of Science, Department of

Mathematics, University of Hail,

Hail-2440, Saudi Arabia

Full list of author information is

available at the end of the article

\section{Abstract}

Several new inequalities for differentiable co-ordinated convex and concave functions in two variables which are related to the left side of Hermite- Hadamard type inequality for co-ordinated convex functions in two variables are obtained.

Mathematics Subject Classification (2000): 26A51; 26D15

Keywords: convex function, co-ordinated convex function, Hermite-Hadamard?'?s inequality, Jensen?'?s integral inequality

\section{Introduction}

The following definition is well known in literature:

A function $f: I \rightarrow \mathbb{R}, \emptyset \neq I \subseteq \mathbb{R}$, is said to be convex on $I$ if the inequality

$$
f(\lambda x+(1-\lambda) y) \leq \lambda f(x)+(1-\lambda) f(y),
$$

holds for all $x, y \in I$ and $\lambda \in[0,1]$.

Many important inequalities have been established for the class of convex functions, but the most famous is the Hermite-Hadamard's inequality (see for instance [1]). This double inequality is stated as:

$$
f\left(\frac{a+b}{2}\right) \leq \frac{1}{b-a} \int_{a}^{b} f(x) d x \leq \frac{f(a)+f(b)}{2}
$$

where $f: I \rightarrow \mathbb{R}, 0 \neq I \subseteq \mathbb{R}$ a convex function, $a, b \in I$ with $a<b$. The inequalities in (1.1) are in reversed order if $f$ is a concave function.

The inequalities (1.1) have become an important cornerstone in mathematical analysis and optimization and many uses of these inequalities have been discovered in a variety of settings. Moreover, many inequalities of special means can be obtained for a particular choice of the function $f$. Due to the rich geometrical significance of Hermite-Hadamard's inequality (1.1), there is growing literature providing its new proofs, extensions, refinements and generalizations, see for example [2-5] and the references therein.

Let us consider now a bidimensional interval $\Delta=:[a, b] \times[c, d]$ in $\mathbb{R}^{2}$ with $a<b$ and $c<d$, a mapping $f: \Delta \rightarrow \mathbb{R}$ is said to be convex on $\Delta$ if the inequality

$$
f(\lambda x+(1-\lambda) z, \lambda y+(1-\lambda) w) \leq \lambda f(x, y)+(1-\lambda) f(z, w),
$$

(c) 2012 Latif and Dragomir; licensee Springer. This is an Open Access article distributed under the terms of the Creative Commons Attribution License (http://creativecommons.org/licenses/by/2.0), which permits unrestricted use, distribution, and reproduction in any medium, provided the original work is properly cited. 
holds for all $(x, y),(z, w) \in \Delta$ and $\lambda \in[0,1]$.

A modification for convex functions on $\Delta$, which are also known as co-ordinated convex functions, was introduced by Dragomir [6,7] as follows:

A function $f: \Delta \rightarrow \mathbb{R}$ is said to be convex on the co-ordinates on $\Delta$ if the partial mappings $f_{y}:[a, b] \rightarrow \mathbb{R}, f_{y}(u)=f(u, y)$ and $f_{x}:[c, d] \rightarrow \mathbb{R}, f_{x}(v)=f(x, v)$ are convex where defined for all $x \in[a, b], y \in[c, d]$.

A formal definition for co-ordinated convex functions may be stated as follows:

Definition 1. [8]A function $f: \Delta \rightarrow \mathbb{R}$ is said to be convex on the co-ordinates on $\Delta$ if the inequality

$$
\begin{aligned}
& f(t x+(1-t) y, s u+(1-s) w) \\
& \quad \leq t s f(x, u)+t(1-s) f(x, w)+s(1-t) f(y, u)+(1-t)(1-s) f(y, w),
\end{aligned}
$$

holds for all $t, s \in[0,1]$ and $(x, u),(y, w) \in \Delta$.

Clearly, every convex mapping $f: \Delta \rightarrow \mathbb{R}$ is convex on the co-ordinates. Furthermore, there exists co-ordinated convex function which is not convex, (see for example [6,7]). For recent results on co-ordinated convex functions we refer the interested reader to [6,8-13].

The following Hermite-Hadamrd type inequality for co-ordinated convex functions on the rectangle from the plane $\mathbb{R}^{2}$ was also proved in [6]:

Theorem 1. [6] Suppose that $f: \Delta \rightarrow \mathbb{R}$ is co-ordinated convex on $\Delta$. Then one has the inequalities:

$$
\begin{aligned}
& f\left(\frac{a+b}{2}, \frac{c+d}{2}\right) \\
& \leq \frac{1}{2}\left[\frac{1}{b-a} \int_{a}^{b} f\left(x, \frac{c+d}{2}\right) d x+\frac{1}{d-c} \int_{c}^{d} f\left(\frac{a+b}{2}, y\right) d y\right] \\
& \leq \frac{1}{(b-a)(d-c)} \int_{a}^{b} \int_{c}^{d} f(x, y) d y d x \\
& \leq \frac{1}{4}\left[\frac{1}{b-a} \int_{a}^{b} f(x, c) d x+\frac{1}{b-a} \int_{a}^{b} f(x, d) d x\right. \\
& \left.+\frac{1}{d-c} \int_{c}^{d} f(a, y) d y+\frac{1}{d-c} \int_{c}^{d} f(b, y) d y\right] \\
& \leq \frac{f(a, c)+f(a, d)+f(b, c)+f(b, d)}{4} .
\end{aligned}
$$

The above inequalities are sharp.

In a recent article [13], Sarikaya et al. proved some new inequalities that give estimate of the difference between the middle and the rightmost terms in (1.2) for differentiable co-ordinated convex functions on rectangle from the plane $\mathbb{R}^{2}$. Motivated by notion given in [13], in the present article, we prove some new inequalities which give estimate between the middle and the leftmost terms in (1.2) for differentiable co-ordinated convex functions on rectangle from the plane $\mathbb{R}^{2}$.

\section{Main results}

The following lemma is necessary and plays an important role in establishing our main results: 
Lemma 1. Let $f: \Delta \subseteq \mathbb{R}^{2} \rightarrow \mathbb{R}$ be a partial differentiable mapping on $\Delta:=[a, b] \times[c$, d] with $a<b, c<d$. If $\frac{\partial^{2} f}{\partial s \partial t} \in L(\Delta)$, then the following identity holds:

$$
\begin{aligned}
& \frac{1}{(b-a)(d-c)} \int_{a}^{b} \int_{c}^{d} f(x, y) d y d x+f\left(\frac{a+b}{2}, \frac{c+d}{2}\right) \\
& -\frac{1}{b-a} \int_{a}^{b} f\left(x, \frac{c+d}{2}\right) d x-\frac{1}{d-c} \int_{c}^{d} f\left(\frac{a+b}{2}, y\right) d y \\
& =(b-a)(d-c) \int_{0}^{1} \int_{0}^{1} K(t, s) \frac{\partial^{2}}{\partial s \partial t} f(t a+(1-t) b, s c+(1-s) d) d s d t
\end{aligned}
$$

where

$$
K(t, s)= \begin{cases}t s, & (t, s) \in\left[0, \frac{1}{2}\right] \times\left[0, \frac{1}{2}\right] \\ t(s-1), & (t, s) \in\left[0, \frac{1}{2}\right] \times\left(\frac{1}{2}, 1\right] \\ s(t-1), & (t, s) \in\left(\frac{1}{2}, 1\right] \times\left[0, \frac{1}{2}\right] \\ (t-1)(s-1), & (t, s) \in\left(\frac{1}{2}, 1\right] \times\left(\frac{1}{2}, 1\right]\end{cases}
$$

Proof. Since

$$
\begin{aligned}
& (b-a)(d-c) \int_{0}^{1} \int_{0}^{1} K(t, s) \frac{\partial^{2}}{\partial s \partial t} f(t a+(1-t) b, s c+(1-s) d) d s d t \\
& =(b-a)(d-c) \int_{0}^{\frac{1}{2}} \int_{0}^{\frac{1}{2}} t s \frac{\partial^{2}}{\partial s \partial t} f(t a+(1-t) b, s c+(1-s) d) d s d t \\
& +(b-a)(d-c) \int_{0}^{\frac{1}{2}} \int_{1}^{1} t(s-1) \frac{\partial^{2}}{\partial s \partial t} f(t a+(1-t) b, s c+(1-s) d) d s d t \\
& +(b-a)(d-c) \int_{1}^{\frac{1}{2}} \int_{0}^{\frac{1}{2}} s(t-1) \frac{\partial^{2}}{\partial s \partial t} f(t a+(1-t) b, s c+(1-s) d) d s d t \\
& +(b-a)(d-c) \int_{1}^{1} \int_{1}^{1}(t-1)(s-1) \frac{\partial^{2}}{\partial s \partial t} f(t a+(1-t) b, s c+(1-s) d) d s d t \\
& \frac{1}{2} \frac{1}{2} \\
& =I_{1}+I_{2}+I_{3}+I_{4} .
\end{aligned}
$$


Now by integration by parts, we have

$$
\begin{aligned}
I_{1} & =(b-a)(d-c) \int_{0}^{\frac{1}{2}} t\left[\int_{0}^{\frac{1}{2}} s \frac{\partial^{2}}{\partial s \partial t} f(t a+(1-t) b, s c+(1-s) d) d s\right] d t \\
& =\frac{1}{4} f\left(\frac{a+b}{2}, \frac{c+d}{2}\right)-\frac{1}{2} \int_{0}^{\frac{1}{2}} f\left(t a+(1-t) b, \frac{c+d}{2}\right) d t \\
- & \frac{1}{2} \int_{0}^{\frac{1}{2}} f\left(\frac{a+b}{2}, s c+(1-s) d\right) d s+\int_{0}^{\frac{1}{2}} \frac{1}{2} f(t a+(1-t) b, s c+(1-s) d) d s d t .
\end{aligned}
$$

If we make use of the substitutions $x=t a+(1-t) b$ and $y=s c+(1-s) d,(t, s) \in[0$, $1]^{2}$, in (2.3), we observe that

$$
\begin{aligned}
I_{1} & =\frac{1}{4} f\left(\frac{a+b}{2}, \frac{c+d}{2}\right)-\frac{1}{2(b-a)} \int_{\frac{a+b}{2} f\left(x, \frac{c+d}{2}\right) d x}^{b} \\
& -\frac{1}{2(d-c)} \int_{\frac{c+d}{2}}^{d} f\left(\frac{a+b}{2}, y\right) d y+\frac{1}{(b-a)(d-c)} \int_{\frac{a+b}{2}}^{b} \int_{\frac{c+d}{2}}^{d} f(x, y) d y d x .
\end{aligned}
$$

Similarly, by integration by parts, we also have that

$$
\begin{aligned}
& I_{2}=\frac{1}{4} f\left(\frac{a+b}{2}, \frac{c+d}{2}\right)-\frac{1}{2(b-a)} \int^{b} f\left(x, \frac{c+d}{2}\right) d x \\
& \frac{a+b}{2} \\
& -\frac{1}{2(d-c)} \int_{c}^{\frac{c+d}{2}} f\left(\frac{a+b}{2}, y\right) d y+\frac{1}{(b-a)(d-c)} \int_{\frac{a+b}{2}}^{b} \int_{c}^{\frac{c+d}{2}} f(x, y) d y d x \\
& I_{3}=\frac{1}{4} f\left(\frac{a+b}{2}, \frac{c+d}{2}\right)-\frac{1}{2(b-a)} \int_{a}^{\frac{a+b}{2}} f\left(x, \frac{c+d}{2}\right) d x \\
& -\frac{1}{2(d-c)} \int_{\frac{c+d}{2}}^{d} f\left(\frac{a+b}{2}, y\right) d y+\frac{1}{(b-a)(d-c)} \int_{a}^{\frac{a+b}{2}} \int_{\frac{c+d}{2}}^{d} f(x, y) d y d x
\end{aligned}
$$


and

$$
\begin{gathered}
I_{4}=\frac{1}{4} f\left(\frac{a+b}{2}, \frac{c+d}{2}\right)-\frac{1}{2(b-a)} \int_{a}^{\frac{a+b}{2}} f\left(x, \frac{c+d}{2}\right) d x \\
-\frac{1}{2(d-c)} \int_{c}^{\frac{c+d}{2}} f\left(\frac{a+b}{2}, y\right) d y+\frac{1}{\frac{a+b}{2} \frac{c+d}{2}} \int_{a}^{2} f(x, y) d y d x .
\end{gathered}
$$

Substitution of the $I_{1}, I_{2}, I_{3}$, and $I_{4}$ in (2.2) gives the desired identity (2.1).

Theorem 2. Let $f: \Delta \subseteq \mathbb{R}^{2} \rightarrow \mathbb{R}$ be a partial differentiable mapping on $\Delta:=[a, b] \times[c$, d] with $a<b, c<d$. If $\left|\frac{\partial^{2} f}{\partial s \partial t}\right|$ is convex on the co-ordinates on $\Delta$, then the following inequality holds:

$$
\begin{aligned}
& \left|\frac{1}{(b-a)(d-c)} \int_{a}^{b} \int_{c}^{d} f(x, y) d y d x+f\left(\frac{a+b}{2}, \frac{c+d}{2}\right)-A\right| \\
& \leq \frac{(b-a)(d-c)}{16}\left[\frac{\left|\frac{\partial^{2}}{\partial s \partial t} f(a, c)\right|+\left|\frac{\partial^{2}}{\partial s \partial t} t(a, d)\right|+\left|\frac{\partial^{2}}{\partial s \partial t} f(b, c)\right|+\left|\frac{\partial^{2}}{\partial s \partial t} f(b, d)\right|}{4}\right]
\end{aligned}
$$

where

$$
A=\frac{1}{b-a} \int_{a}^{b} f\left(x, \frac{c+d}{2}\right) d x+\frac{1}{d-c} \int_{c}^{d} f\left(\frac{a+b}{2}, y\right) d y
$$

Proof. From Lemma 1, we have

$$
\begin{aligned}
& \left|\frac{1}{(b-a)(d-c)} \int_{a}^{b} \int_{c}^{d} f(x, y) d y d x+f\left(\frac{a+b}{2}, \frac{c+d}{2}\right)-A\right| \\
& \leq(b-a)(d-c) \int_{0}^{1} \int_{0}^{1}|K(t, s)|\left|\frac{\partial^{2}}{\partial s \partial t} f(t a+(1-t) b, s c+(1-s) d)\right| d s d t
\end{aligned}
$$

Since $\left|\frac{\partial^{2} f}{\partial s \partial t}\right|$ is convex on the co-ordinates on $\Delta$, we have

$$
\begin{aligned}
& \left|\frac{\partial^{2}}{\partial s \partial t} f(t a+(1-t) b, s c+(1-s) d)\right| \leq t s\left|\frac{\partial^{2}}{\partial s \partial t} f(a, c)\right|+t(1-s)\left|\frac{\partial^{2}}{\partial s \partial t} f(a, d)\right| \\
& +s(1-t)\left|\frac{\partial^{2}}{\partial s \partial t} f(b, c)\right|+(1-t)(1-s)\left|\frac{\partial^{2}}{\partial s \partial t} f(b, d)\right| .
\end{aligned}
$$


Substitution of (2.6) in (2.5) gives the following inequality:

$$
\begin{aligned}
& \left|\frac{1}{(b-a)(d-c)} \int_{a}^{b} \int_{c}^{d} f(x, y) d y d x+f\left(\frac{a+b}{2}, \frac{c+d}{2}\right)-A\right| \\
& \leq(b-a)(d-c) \int_{0}^{1} \int_{0}^{1}|K(t, s)|\left[\left|\frac{\partial^{2}}{\partial s \partial t} f(a, c)\right| t s+\left|\frac{\partial^{2}}{\partial s \partial t} f(a, d)\right| t(1-s)\right. \\
& \left.+\left|\frac{\partial^{2}}{\partial s \partial t}(b, c)\right| s(1-t)+\left|\frac{\partial^{2}}{\partial s \partial t}(b, d)\right|(1-t)(1-s)\right] d s d t=(b-a)(d-c) \\
& \times\left\{\int _ { 0 } ^ { \frac { 1 } { 2 } } \frac { 1 } { 2 } \int _ { 0 } ^ { t } \left[\left|\frac{\partial^{2}}{\partial s \partial t}(a, c)\right| t s+\left|\frac{\partial^{2}}{\partial s \partial t}(a, d)\right| t(1-s)+\left|\frac{\partial^{2}}{\partial s \partial t} f(b, c)\right| s(1-t)\right.\right. \\
& \left.+\left|\frac{\partial^{2}}{\partial s \partial t} f(b, d)\right|(1-t)(1-s)\right] d s d t+\int_{0}^{\frac{1}{2}} \frac{1}{2} t(1-s)\left[\left|\frac{\partial^{2}}{\partial s \partial t} f(a, c)\right| t s\right. \\
& \left.+\left|\frac{\partial^{2}}{\partial s \partial t} f(b, d)\right|(1-t)(1-s)\right] d s d t+\int_{0}^{1} \frac{1}{2} \frac{1}{2} \frac{1}{2} s(1-t)(1-s)\left[\left|\frac{\partial^{2}}{\partial s \partial t} f(a, c)\right| t s+\right. \\
& \left.+\left|\frac{\partial^{2}}{\partial s \partial t} f(a, d)\right| t(1-s)+\left|\frac{\partial^{2}}{\partial s \partial t} f(b, c)\right| s(1-t)+\left|\frac{\partial^{2}}{\partial s \partial t} f(b, d)\right|(1-t)(1-s)\right] d s d t \\
& +\frac{1}{\partial s \partial t} f(a, c)|t s+| \frac{\partial^{2}}{\partial s \partial t} f(a, d)|t(1-s)+| \frac{\partial^{2}}{\partial s \partial t} f(b, c) \mid s(1-t)
\end{aligned}
$$

Evaluating each integral in (2.7) and simplifying, we get (2.4). Hence the proof of the theorem is complete.

Theorem 3. Let $f: \Delta \subseteq \mathbb{R}^{2} \rightarrow \mathbb{R}$ be a partial differentiable mapping on $\Delta:=[a, b] \times$ $[c, d]$ with $a<b, c<d$. If $\left|\frac{\partial^{2} f}{\partial s \partial t}\right|^{q}$ is convex on the co-ordinates on $\Delta$ and $p, q>1$, $\frac{1}{p}+\frac{1}{q}=1$, then the following inequality holds:

$$
\begin{aligned}
& \left|\frac{1}{(b-a)(d-c)} \int_{a}^{b} \int_{a}^{d} f(x, y) d y d x+f\left(\frac{a+b}{2}, \frac{c+d}{2}\right)-A\right| \\
& \leq \frac{(b-a)(d-c)}{4(p+1)^{\frac{2}{p}}}\left[\frac{\left|\frac{\partial^{2}}{\partial s \partial t}(a, c)\right|^{q}+\left|\frac{\partial^{2}}{\partial s \partial t}(a, d)\right|^{q}+\left|\frac{\partial^{2}}{\partial s \partial t}(b, c)\right|^{q}+\left|\frac{\partial^{2}}{\partial s \partial t}(b, d)\right|^{q}}{4}\right]^{\frac{1}{q}},
\end{aligned}
$$

where $A$ is as given in Theorem 2. 
Proof. From Lemma 1, we have

$$
\begin{aligned}
& \left|\frac{1}{(b-a)(d-c)} \int_{a}^{b} \int_{c}^{d} f(x, y) d y d x+f\left(\frac{a+b}{2}, \frac{c+d}{2}\right)-A\right| \\
& \leq(b-a)(d-c) \int_{0}^{1} \int_{0}^{1}|K(t, s)|\left|\frac{\partial^{2}}{\partial s \partial t} f(t a+(1-t) b, s c+(1-s) d)\right| d s d t .
\end{aligned}
$$

Now using the well-known Hölder inequality for double integrals, we obtain

$$
\begin{aligned}
& \int_{0}^{1} \int_{0}^{1}|K(t, s)|\left|\frac{\partial^{2}}{\partial s \partial t} f(t a+(1-t) b, s c+(1-s) d)\right| d s d t \\
& \leq\left(\int_{0}^{1} \int_{0}^{1}|K(t, s)|^{p} d s d t\right)^{\frac{1}{p}}\left(\int_{0}^{1} \int_{0}^{1}\left|\frac{\partial^{2}}{\partial s \partial t} f(t a+(1-t) b, s c+(1-s) d)\right|^{q} d s d t\right)^{\frac{1}{q}}
\end{aligned}
$$

Since $\left|\frac{\partial^{2} f}{\partial s \partial t}\right|^{q}$ is convex on the co-ordinates on $\Delta$, we have

$$
\begin{aligned}
& \int_{0}^{1} \int_{0}^{1}\left|\frac{\partial^{2}}{\partial s \partial t} f(t a+(1-t) b, s c+(1-s) d)\right|^{q} d s d t \\
& \leq \int_{0}^{1} \int_{0}^{1}\left[t s\left|\frac{\partial^{2}}{\partial s \partial t} f(a, c)\right|^{q}+t(1-s)\left|\frac{\partial^{2}}{\partial s \partial t} f(a, d)\right|^{q}\right. \\
& \left.+s(1-t)\left|\frac{\partial^{2}}{\partial s \partial t} f(b, c)\right|^{q}+(1-t)(1-s)\left|\frac{\partial^{2}}{\partial s \partial t} f(b, d)\right|^{q}\right] d s d t \\
& =\frac{\left|\frac{\partial^{2}}{\partial s \partial t} f(a, c)\right|^{q}+\left|\frac{\partial^{2}}{\partial s \partial t} f(a, d)\right|^{q}+\left|\frac{\partial^{2}}{\partial s \partial t} f(b, c)\right|^{q}+\left|\frac{\partial^{2}}{\partial s \partial t} f(b, d)\right|^{q}}{4} .
\end{aligned}
$$

Also, we notice that

$$
\begin{aligned}
\int_{0}^{1} \int_{0}^{1}|K(t, s)|^{p} d s d t & =\int_{0}^{\frac{1}{2}} \int_{0}^{\frac{1}{2}} t^{p} s^{p} d s d t+\int_{0}^{\frac{1}{2}} \int_{\frac{1}{2}}^{1} t^{p}(1-s)^{p} d s d t \\
& +\int_{0}^{\frac{1}{2}} \int_{0}^{\frac{1}{2}} s^{p}(1-t)^{p} d s d t+\int_{\frac{1}{2}}^{1} \frac{1}{2}(1-t)^{p}(1-s)^{p} d s d t \\
& =\frac{4}{(p+1)^{2}}\left(\frac{1}{2}\right)^{2(p+1)} \cdot
\end{aligned}
$$


Using (2.11) and (2.12) in (2.10), we obtain

$$
\begin{aligned}
& \int_{0}^{1} \int_{0}^{1}|K(t, s)|\left|\frac{\partial^{2}}{\partial s \partial t} f(t a+(1-t) b, s c+(1-s) d)\right| d s d t \\
& \leq \frac{1}{4(p+1) \frac{2}{p}}\left[\frac{\left|\frac{\partial^{2}}{\partial s \partial t} f(a, c)\right|^{q}+\left|\frac{\partial^{2}}{\partial s \partial t} f(a, d)\right|^{q}+\left|\frac{\partial^{2}}{\partial s \partial t} f(b, c)\right|^{q}+\left|\frac{\partial^{2}}{\partial s \partial t} f(b, d)\right|^{q}}{4}\right]^{\frac{1}{q}} .
\end{aligned}
$$

Utilizing the last inequality in (2.9) gives us (2.8). This completes the proof of the theorem.

Now we state our next result in:

Theorem 4. Let $f: \Delta \subseteq \mathbb{R}^{2} \rightarrow \mathbb{R}$ be a partial differentiable mapping on $\Delta:=[a, b] \times$ $[c, d]$ with $a<b, c<d$. If $\left|\frac{\partial^{2} f}{\partial s \partial t}\right|^{q}$ is convex on the co-ordinates on $\Delta$ and $q \geq 1$, then the following inequality holds:

$$
\begin{aligned}
& \left|\frac{1}{(b-a)(d-c)} \int_{a}^{b} \int_{c}^{d} f(x, y) d y d x+f\left(\frac{a+b}{2}, \frac{c+d}{2}\right)-A\right| \\
& \leq \frac{(b-a)(d-c)}{16}\left[\frac{\left|\frac{\partial^{2}}{\partial s \partial t}(a, c)\right|^{q}+\left|\frac{\partial^{2}}{\partial s \partial t}(a, d)\right|^{q}+\left|\frac{\partial^{2}}{\partial s \partial t}(b, c)\right|^{q}+\left|\frac{\partial^{2}}{\partial s \partial t}(b, d)\right|^{q}}{4}\right]^{\frac{1}{q}},
\end{aligned}
$$

where $A$ is as given in Theorem 2.

Proof. By using Lemma 1, we have that the following inequality:

$$
\begin{aligned}
& \left|\frac{1}{(b-a)(d-c)} \int_{a}^{b} \int_{a}^{d} f(x, y) d y d x+f\left(\frac{a+b}{2}, \frac{c+d}{2}\right)-A\right| \\
& \leq(b-a)(d-c) \int_{0}^{1} \int_{0}^{1}|K(t, s)|\left|\frac{\partial^{2}}{\partial s \partial t} f(t a+(1-t) b, s c+(1-s) d)\right| d s d t .
\end{aligned}
$$

By the power mean inequality, we have

$$
\begin{aligned}
& \int_{0}^{1} \int_{0}^{1}|K(t, s)|\left|\frac{\partial^{2}}{\partial s \partial t} f(t a+(1-t) b, s c+(1-s) d)\right| d s d t \\
& \leq\left(\int_{0}^{1} \int_{0}^{1}|K(t, s)| d s d t\right)^{1-\frac{1}{q}} \\
& \times\left(\int_{0}^{1} \int_{0}^{1}|K(t, s)|\left|\frac{\partial^{2}}{\partial s \partial t} f(t a+(1-t) b, s c+(1-s) d)\right|^{q} d s d t\right)^{\frac{1}{q}} \\
& =\left(\frac{1}{16}\right)^{1-\frac{1}{q}\left(\int_{0}^{1} \int_{0}^{1}|K(t, s)|\left|\frac{\partial^{2}}{\partial s \partial t} f(t a+(1-t) b, s c+(1-s) d)\right|^{q} d s d t\right)^{\frac{1}{q}} .} .
\end{aligned}
$$


Using the fact that $\left|\frac{\partial^{2} f}{\partial s \partial t}\right|^{q}$ is convex on the co-ordinates on $\Delta$, we get

$$
\begin{aligned}
& \left|\frac{\partial^{2}}{\partial s \partial t} f(t a+(1-t) b, s c+(1-s) d)\right|^{q} \\
& =t s\left|\frac{\partial^{2}}{\partial s \partial t} f(a, c)\right|^{q}+t(1-s)\left|\frac{\partial^{2}}{\partial s \partial t} f(a, d)\right|^{q}+s(1-t)\left|\frac{\partial^{2}}{\partial s \partial t} f(b, c)\right|^{q} \\
& +(1-t)(1-s)\left|\frac{\partial^{2}}{\partial s \partial t} f(b, d)\right|^{q}
\end{aligned}
$$

and hence, we obtain

$$
\begin{aligned}
& \int_{0}^{1} \int_{0}^{1}|K(t, s)|\left|\frac{\partial^{2}}{\partial s \partial t} f(t a+(1-t) b, s c+(1-s) d)\right|^{q} d s d t \\
& \leq \int_{0}^{1} \int_{0}^{1}|K(t, s)|\left[t s\left|\frac{\partial^{2}}{\partial s \partial t} f(a, c)\right|^{q}+t(1-s)\left|\frac{\partial^{2}}{\partial s \partial t} f(a, d)\right|^{q}\right. \\
& \left.+s(1-t)\left|\frac{\partial^{2}}{\partial s \partial t} f(b, c)\right|^{q}+(1-t)(1-s)\left|\frac{\partial^{2}}{\partial s \partial t} f(b, d)\right|^{q}\right] d s d t \\
& =\frac{1}{64}\left[\left|\frac{\partial^{2}}{\partial s \partial t} f(a, c)\right|^{q}+\left|\frac{\partial^{2}}{\partial s \partial t} f(a, d)\right|^{q}+\left|\frac{\partial^{2}}{\partial s \partial t} f(b, c)\right|^{q}+\left|\frac{\partial^{2}}{\partial s \partial t} f(b, d)\right|^{q}\right] .
\end{aligned}
$$

Therefore (2.15) becomes

$$
\begin{aligned}
& \int_{0}^{1} \int_{0}^{1}|K(t, s)|\left|\frac{\partial^{2}}{\partial s \partial t} f(t a+(1-t) b, s c+(1-s) d)\right| d s d t \\
& \leq \frac{1}{16}\left[\frac{\left|\frac{\partial^{2}}{\partial s \partial t} f(a, c)\right|^{q}+\left|\frac{\partial^{2}}{\partial s \partial t} f(a, d)\right|^{q}+\left|\frac{\partial^{2}}{\partial s \partial t} f(b, c)\right|^{q}+\left|\frac{\partial^{2}}{\partial s \partial t} f(b, d)\right|^{q}}{4}\right]^{\frac{1}{q}}
\end{aligned}
$$

Substitution of (2.16) in (2.14), we obtain (2.13). Hence the proof is complete.

Remark 1. Since $2^{p}>p+1$ if $p>1$ and accordingly

$$
\frac{1}{4}<\frac{1}{2(p+1) \frac{1}{p}}
$$

and hence we have that the following inequality:

$$
\frac{1}{16}<\frac{1}{4} \cdot \frac{1}{4}<\frac{1}{2(p+1) \frac{1}{p}} \cdot \frac{1}{2(p+1) \frac{1}{p}}=\frac{1}{4(p+1) \frac{2}{p}},
$$

and as a consequence we get an improvement of the constant in Theorem 3.

Following theorem is about concave functions on the co-ordinates on $\Delta$ : 
Theorem 5. Let $f: \Delta \subseteq \mathbb{R}^{2} \rightarrow \mathbb{R}$ be a partial differentiable mapping on $\Delta:=[a, b] \times$ $[c, d]$ with $a<b, c<d$. If $\left|\frac{\partial^{2} f}{\partial s \partial t}\right|^{q}$ is concave on the co-ordinates on $\Delta$ and $q \geq 1$, then we have the inequality:

$$
\begin{aligned}
& \left|\frac{1}{(b-a)(d-c)} \int_{a}^{b} \int_{c}^{d} f(x, y) d y d x+f\left(\frac{a+b}{2}, \frac{c+d}{2}\right)-A\right| \\
& \leq \frac{(b-a)(d-c)}{64}\left[\left|\frac{\partial^{2}}{\partial s \partial t} f\left(\frac{a+2 b}{3}, \frac{c+2 d}{3}\right)\right|+\left|\frac{\partial^{2}}{\partial s \partial t} f\left(\frac{a+2 b}{3}, \frac{2 c+d}{3}\right)\right|\right. \\
& \left.\left|\frac{\partial^{2}}{\partial s \partial t} f\left(\frac{2 a+b}{3}, \frac{c+2 d}{3}\right)\right|+\left|\frac{\partial^{2}}{\partial s \partial t} f\left(\frac{2 a+b}{3}, \frac{2 c+d}{3}\right)\right|\right]
\end{aligned}
$$

where $A$ is as defined in Theorem 2.

Proof. By the concavity of $\left|\frac{\partial^{2} f}{\partial s \partial t}\right|^{q}$ on the co-ordinates on $\Delta$ and power mean inequality, we note that the following inequality holds:

$$
\begin{aligned}
\mid \frac{\partial^{2}}{\partial s \partial t} f\left(\lambda x+\left(1-\left.\lambda(y, v)\right|^{q}\right.\right. & \geq \lambda\left|\frac{\partial^{2}}{\partial s \partial t} f(x, v)\right|^{q}+(1-\lambda)\left|\frac{\partial^{2}}{\partial s \partial t} f(y, v)\right|^{q} \\
& \geq\left(\lambda\left|\frac{\partial^{2}}{\partial s \partial t} f(x, v)\right|+(1-\lambda)\left|\frac{\partial^{2}}{\partial s \partial t} f(y, v)\right|\right)^{q}
\end{aligned}
$$

for all $x, y \in[a, b], \lambda \in[0,1]$ and for fixed $v \in[c, d]$.

Hence,

$$
\left|\frac{\partial^{2}}{\partial s \partial t} f(\lambda x+(1-\lambda) y, v)\right| \geq \lambda\left|\frac{\partial^{2}}{\partial s \partial t} f(x, v)\right|+(1-\lambda)\left|\frac{\partial^{2}}{\partial s \partial t} f(y, v)\right|,
$$

for all $x, y \in[a, b], \lambda \in[0,1]$ and for fixed $v \in[c, d]$.

Similarly, we can show that

$$
\left|\frac{\partial^{2}}{\partial s \partial t} f(u, \lambda z+(1-\lambda) w)\right| \geq \lambda\left|\frac{\partial^{2}}{\partial s \partial t} f(u, z)\right|+(1-\lambda)\left|\frac{\partial^{2}}{\partial s \partial t} f(u, w)\right|,
$$

for all $z, w \in[c, d], \lambda \in[0,1]$ and for fixed $u \in[a, d]$, thus $\left|\frac{\partial^{2} f}{\partial s \partial t}\right|$ is concave on the co-ordinates on $\Delta$. 
It is clear from Lemma 1 that

$$
\begin{aligned}
& \left|\frac{1}{(b-a)(d-c)} \int_{a}^{b} \int_{c}^{d} f(x, y) d y d x+f\left(\frac{a+b}{2}, \frac{c+d}{2}\right)-A\right| \\
& \leq(b-a)(d-c) \int_{0}^{1} \int_{0}^{1}|K(t, s)|\left|\frac{\partial^{2}}{\partial s \partial t} f(t a+(1-t) b, s c+(1-s) d)\right| d s d t \\
& =(b-a)(d-c)\left|\int_{0}^{\frac{1}{2}} \frac{1}{2} \int_{0} s t\right| \frac{\partial^{2}}{\partial s \partial t} f(t a+(1-t) b, s c+(1-s) d) \mid d s d t \\
& +\int_{0}^{\frac{1}{2}} \int_{\frac{1}{2}}^{\frac{1}{2}} t(1-s)\left|\frac{\partial^{2}}{\partial s \partial t} f(t a+(1-t) b, s c+(1-s) d)\right| d s d t \\
& +\int_{1}^{1} \int_{0}^{\frac{1}{2}} s(1-t)\left|\frac{\partial^{2}}{\partial s \partial t} f(t a+(1-t) b, s c+(1-s) d)\right| d s d t \\
& \frac{1}{2} \\
& \left.+\int_{1}^{1} \int_{1}^{1}(1-t)(1-s)\left|\frac{\partial^{2}}{\partial s \partial t} f(t a+(1-t) b, s c+(1-s) d)\right| d s d t\right] . \\
& \frac{1}{2} \frac{1}{2}
\end{aligned}
$$

Since $\left|\frac{\partial^{2} f}{\partial s \partial t}\right|$ is concave on the co-ordinates, we have, by Jensen's inequality for integrals, that:

$$
\begin{aligned}
& \frac{1}{2} \frac{1}{2} \\
& \int_{0}^{\frac{1}{2}} \int_{0}^{\frac{1}{2}} s t\left|\frac{\partial^{2}}{\partial s \partial t} f(t a+(1-t) b, s c+(1-s) d)\right| d s d t \\
& =\int_{0}^{\frac{1}{2}} t\left[\int_{0}^{\frac{1}{2}} s\left|\frac{\partial^{2}}{\partial s \partial t} f(t a+(1-t) b, s c+(1-s) d)\right| d s\right] d t \\
& \leq \int_{0}^{\frac{1}{2}} r\left(\int_{0}^{\frac{1}{2}} s d s\right)\left|\frac{\partial^{2}}{\partial s \partial t} f\left(t a+(1-t) b, \frac{\int_{0}^{\frac{1}{2}} s(s c+(1-s) d) d s}{\frac{1}{2} s d s}\right)\right| d t \\
& =\frac{1}{8} \int_{0}^{\frac{1}{2}} t\left|\frac{\partial^{2}}{\partial s \partial t} f\left(t a+(1-t) b, \frac{c+2 d}{3}\right)\right| d t \\
& \leq \frac{1}{8}\left(\int_{0}^{\frac{1}{2}} t d t\right)\left|\frac{\partial^{2}}{\partial s \partial t} f\left(\frac{\int_{0}^{\frac{1}{2}} t(t a+(1-t) b) d t}{\frac{1}{2} t d t}, \frac{c+2 d}{3}\right)\right| \\
& =\frac{1}{64}\left|\frac{\partial^{2}}{\partial s \partial t} f\left(\frac{a+2 b}{3}, \frac{c+2 d}{3}\right)\right| \text {. }
\end{aligned}
$$


In a similar way, we also have that

$$
\begin{aligned}
& \int_{0}^{\frac{1}{2}} \int_{\frac{1}{2}}^{1} t(1-s)\left|\frac{\partial^{2}}{\partial s \partial t} f(t a+(1-t) b, s c+(1-s) d)\right| d s d t \\
& \leq \frac{1}{64}\left|\frac{\partial^{2}}{\partial s \partial t} f\left(\frac{a+2 b}{3}, \frac{2 c+d}{3}\right)\right|, \\
& \int_{\frac{1}{2}}^{1} \int_{0}^{\frac{1}{2}} s(1-t)\left|\frac{\partial^{2}}{\partial s \partial t} f(t a+(1-t) b, s c+(1-s) d)\right| d s d t \\
& \leq \frac{1}{64}\left|\frac{\partial^{2}}{\partial s \partial t} f\left(\frac{2 a+b}{3}, \frac{c+2 d}{3}\right)\right|
\end{aligned}
$$

and

$$
\begin{aligned}
& \int_{\frac{1}{2}}^{1} \int_{\frac{1}{2}}^{1}(1-t)(1-s)\left|\frac{\partial^{2}}{\partial s \partial t} f(t a+(1-t) b, s c+(1-s) d)\right| d s d t \\
& \leq \frac{1}{64}\left|\frac{\partial^{2}}{\partial s \partial t} f\left(\frac{2 a+b}{3}, \frac{c+2 d}{3}\right)\right| .
\end{aligned}
$$

By making use of (2.19)-(2.22) in (2.18), we get the desired result. This completes the proof.

\section{Acknowledgements}

This article is in final form and no version of it will be submitted for publication elsewhere.

\section{Author details}

${ }^{1}$ College of Science, Department of Mathematics, University of Hail, Hail-2440, Saudi Arabia ${ }^{2}$ School of Engineering \& Science, Victoria University, PO Box 14428, Melbourne City, MC 8001, Australia

\section{Authors' contributions}

MAL and SSD carried out the design of the study and performed the analysis. Both of the authors read and approved the final version of the manuscript.

\section{Competing interests}

The authors declare that they have no competing interests.

Received: 13 October 2011 Accepted: 15 February 2012 Published: 15 February 2012

\section{References}

1. Pečarić, JE, Proschan, F, Tong, YL: Convex Functions, Partial Ordering and Statistical Applications. Academic Press, New York (1991)

2. Alomari, M, Darus, M, Dragomir, SS: Inequalities of Hermite-Hadamard's type for functions whose derivatives absolute values are quasi-convex. RGMIA Res Rep Collect. 12(suppl. 14) (2009)

3. Dragomir, SS, Agarwal, RP: Two inequalities for differentiable mappings and applications to special means of real numbers and to Trapezoidal formula. Appl Math Lett. 11(5):91-95 (1998). doi:10.1016/S0893-9659(98)00086-X

4. Kirmaci, US: Inequalities for differentiable mappings and applications to special means of real numbers to midpoint formula. Appl Math Comput. 147, 137-146 (2004). doi:10.1016/50096-3003(02)00657-4

5. Pearce, CME, Pečarić, JE: Inequalities for differentiable mappings with applications to special means and quadrature formula. Appl Math Lett. 13, 51-55 (2000) 
6. Dragomir, SS: On Hadamard's inequality for convex functions on the co-ordinates in a rectangle from the plane. Taiwanese J Math. 4, 775-788 (2001)

7. Dragomir, SS, Pearce, CEM: Selected Topics on Hermite-Hadamard Inequalities and Applications. RGMIA Monographs, Victoria University (2000) Online: http://www.staff.vu.edu.au/RGMIA/monographs/hermite_hadamard.html

8. Latif, MA, Alomari, M: Hadamard-type inequalities for product two convex functions on the co-ordinetes. Int Math Forum. 4(47):2327-2338 (2009)

9. Alomari, M, Darus, M: Co-ordinated s-convex function in the first sense with some Hadamard-type inequalities. Int Contemp Math Sci. 3(32):1557-1567 (2008)

10. Alomari, M, Darus, M: On the Hadamard's inequality for log-convex functions on the coordinates. J Inequal Appl 13 (2009). (Article ID 283147)

11. Latif, MA, Alomari, M: On the Hadamard-type inequalities for h-convex functions on the co-ordinetes. Int J Math Anal. 3(33):1645-1656 (2009)

12. Özdemir, ME, Set, E, Sarikaya, MZ: New some Hadamard's type inequalities for coordinated $m$-convex and $(a, m)$-convex functions. RGMIA Res Rep Collect 13 (2010). Supplement, (Article 4)

13. Sarikaya, MZ, Set, E, Özdemir, ME, Dragomir, SS: Some new Hadamard's type inequalities for co-ordinated convex functions. arXiv:1005.0700v1 [math.CA]

doi:10.1186/1029-242X-2012-28

Cite this article as: Latif and Dragomir: On some new inequalities for differentiable co-ordinated convex

functions. Journal of Inequalities and Applications 2012 2012:28.

\section{Submit your manuscript to a SpringerOpen ${ }^{\circ}$} journal and benefit from:

- Convenient online submission

- Rigorous peer review

- Immediate publication on acceptance

- Open access: articles freely available online

- High visibility within the field

- Retaining the copyright to your article

Submit your next manuscript at $\boldsymbol{s p r i n g e r o p e n . c o m ~}$ 